\title{
Business Intelligence: Concepts, Components, Features, Extraction Transformation Loading Technology
}

\author{
Saumya Patel \\ Assistant Professor \\ Cmpica, Charusat, Changa
}

\begin{abstract}
The ERP systems, CRM systems, banking and credit card systems and Corporate Governance regulations have exponentially increased data volumes needing analysis.Data storing mechanism and strong solutions would be needed for the entire process of data extraction from its sources to its analysis. Because of constant innovation in data processing, huge volume of data is needed to be stored in more detailed format. To analyse the data meaningfully, data reduction and a well-defined structured is also needed.The analysis necessary to create "business intelligence" from the collected raw data requires a varied tool set. By investigating the business needs, it defines the abilities on the roles and requirements of real time BI. The paper explores the concept of BI, objective of BI system, its key components and features, and Extraction, Transformation, Loading (ETL) process.
\end{abstract}

\section{Keywords}

Business Intelligence, SAP, ETL, NetWeaver, BI

\section{INTRODUCTION}

[1]To gather, store, analyse and provide the access to the data Business Intelligence provides different category of applications and technology which ultimately helps the enterprise user in making better business decisions. BI applications contain decision support system, statistical analysis, online analytical processing (OLAP), forecasting, Data Mining and activities of query and reporting.

The enterprise users and decision makers of globally operating systems realize that their strength is to use the effective information. Making effective use of information is tremendously difficult because the information exists in in many systems and in some cases in many countries. With the help of Business Intelligence systems these challenges can be met.

A particular challenge is to extract and prepare consolidated transaction data from mySAP Business Suite applications and source systems from other providers in heterogeneous system landscapes.Detailed level data analysis and multimedia presentation options are required along with integrated data collection processwhen business demand for the high-quality business information. To incorporate all these features and enabling the need for real-time data collection the increasing demand for Business Intelligence solutions is enormous.

[2]defined BIthat "To permit strategic and operational decision makers to cease their actions according to the company strategy and supervise the time-critical operational process, it should contain data warehouse and a reactive component."A Data Warehouse can help to organize the data. It brings together all operative DataSources (these are mostly heterogeneous and have differing degrees of detail). The job of the warehouse is to provide this data in a usable form to the whole organization.[1]So in summary, Business Intelligence software is the collection of applications needed to make sense of business data. The Data Warehouse, a component of this Business Intelligence tool set, is the more specific tool responsible for the cleanup, loading, and storage of the data needed by the business.

\section{BUSINESS INTELLIGENCE SYSTEMS OBJECTIVES}

Standardized structuring and display of all business information: It is essential for decision makers to get the trustworthy information from purchasing, sales and distribution, production, finance and human resource departments. Broad picture of each business area as a whole and an up-to-date system is also needed. The data is stored and defined distinctively across the entire organization which will help avoiding arising errors but it leads to the high demand of the data collection process from the underlying DataSources.

Simple access to business information via a single point of entry: To call up the information from the central point, information must be combined evenly and consistently. Due to this, to provide the vital services, modern Data Warehouse generally demands a separate database which enables a standalone application environment.

Highly developed reporting for analysis with self-service for all areas:Competent analysis and significant multimedia visualization techniques are essential in terms of presentation.

Quick and cost-efficient implementation: The straightforward loading of classified data and integration of Data Warehouse into an OLTP system gives a prominent cost factor. Along with this, delivered business-based Business Intelligence content and metadata management also has a domineering role here.

High performance environment. Data modeling from heterogeneous sources: Without integrating heterogeneous sources, Data analysis cannot be performed through Data Warehouse.Scheduling tools are needed for the data load in a distinct batch jobs at performance-friendly times.

Relieving OLTP systems: A separate Data Warehouse sever allows you to do the data analysis elsewhere. Earlier, OLTP systems were overly burdened by heavy storage of data and performing data analysis at the same time. 


\section{Business Explorer Suite (BEx)}

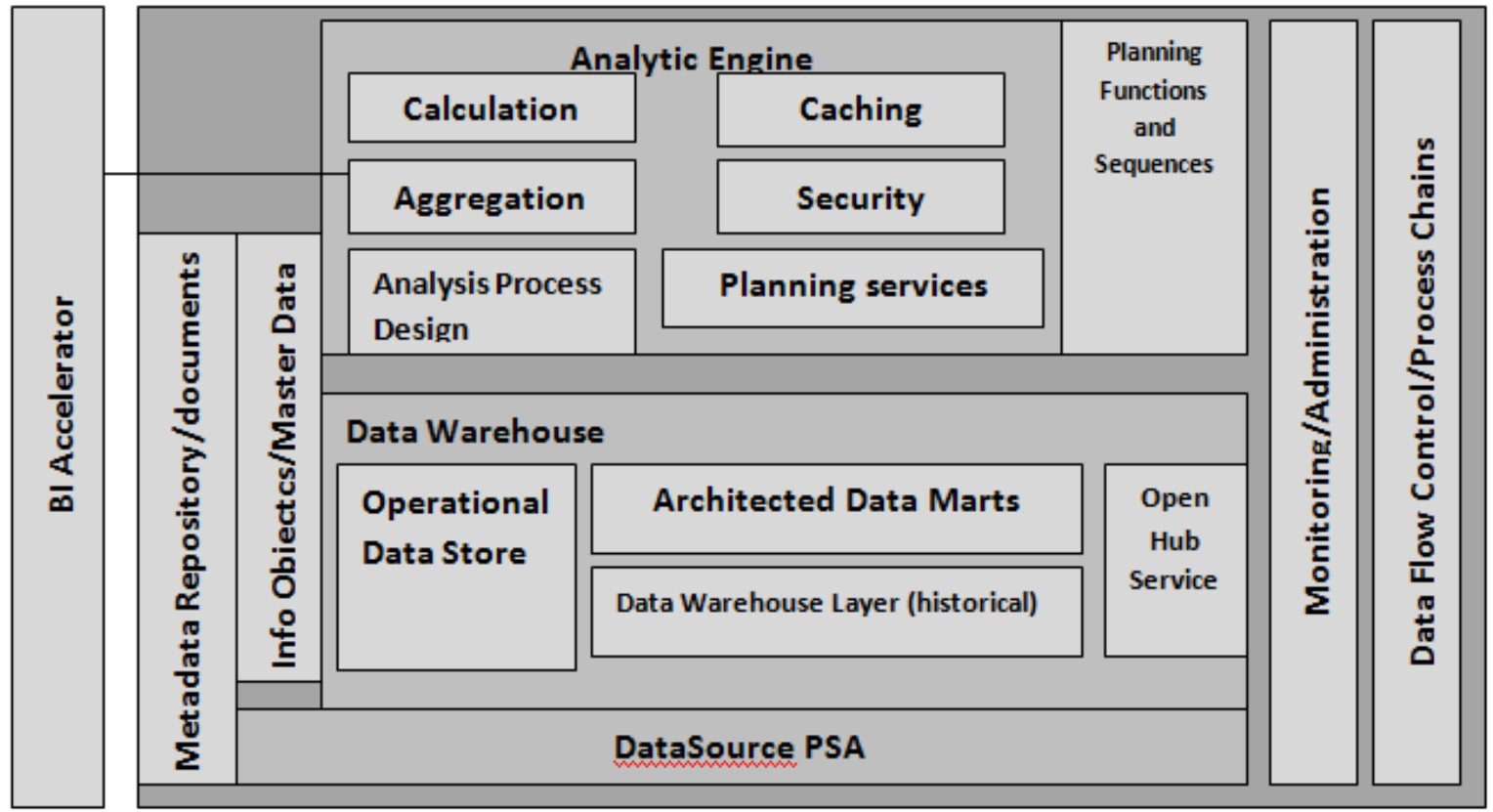

Figure 1: BI Architecture- Platform and Data Warehouse

\section{KEY COMPONENTS AND FEATURES OF BI}

To enable businesses to conquer the maximum value from the information they collect, a core component of SAP NetWeaver, BI provides a suite of Business Intelligence tools, Data Warehousing functionality and a Business Intelligence platform. To evaluate and interpret the data, BI also provides the flexible reporting and analysis tools which will perform the data distribution as well.

a) BI Suite/Business Explorer (BEx): [3]For strong decision making support and tactical analysis within a business, the SAP NetWeaver Business Intelligence Suite, SAP Business Explorer (SAP BEx) provides the flexible reporting and analysis tools. The tools include reporting, query and analysis functions though which at various levels historical or current data evaluation can be done using different perspective. SAP Business Explorer tools can also be used to create planning applications, and for planning and data entry in BW Integrated Planning. Many outputs options are supported to the BEx tool, including formatted Microsoft Excel, Web cockpits, formatted Web output (BEx Reports) and Adobe PDF documents.

Data can beanalysed in the following areas of Business Explorer:

- BExAnalyzer (Microsoft Excel-based analysis tool with pivot-table-like features)

- BEx Web Analyzer (Web-based analysis tool with pivot-table-like features)

- BEx Web Application Designer (customerdefined and SAP BI Content provided)

- $\quad$ BEx Report Designer (highly formatted Web output) b) BEx Web Application Designer: [4]BEx Web Application Designer is a desktop application for creating Web applications to generate HTML pages that contain BI-specific content such as various charts, maps and tables. Web application can be saved as URL and can be accessible from the Internet, intranet or mobile devices. In Web Application Designer, Web Application Wizard has been integrated which helps in creating Web applications.

c) Enterprise Reporting:Positioning control and display formatting can be accomplished in several ways in BI with Enterprise reporting. [5]Using BExAnalyzer the query outputs can be analysed and it is also possible to create reports, charts and graphics. All the functions provided by Microsoft Excel can also be used because Excel is integrated with BExAnalyzer. To access physical BI data, third party tool can be used and it is also possible to access the data residing physically on other system as well.

d) Information broadcasting: [6]To distribute the reporting results to different audiences and the users who are not regular BI users, Business Explorer provides the Information Broadcasting function. Using this function, broadcasting can be done as one-time event, regular scheduled event, or also used just to alert personnel of any serious situation which needs attention. The distribution can be scheduled via Webbased UI.

e) The BI Platform: [7]The BI Platform provides various analytical technologies, function and functions as the technological infrastructure. The BI services in BI Platform Layer supports the complex analysis tasks and functions. With the help of Analytical Engine data can 
be processed and it also does the data manipulation as a part of BI Integrated Planning in BEx analysis navigations. The discrete analysis tools such as the Analysis Process Designer (APD) and the Data Mining provide the analysts at your company with the tools to merge, mine, pre-process, store, and analyse data without support from the technical team.

f) Mobile Reporting: The Web applications created with Web Application Designer, can be called using BEx Mobile Intelligence. [1]The following devices are supported:

- Personal digital assistant (PDA) with Windows CE 3.0and Pocket Internet Explorer

- WAP-enabled mobile telephone

- i-Mode-enabled mobile telephone

- Mobile device with EPOC32 operating system (the Nokia Communicator 9210, for example)

\section{ETL TECHNOLOGY}

[8] ETL stands for Extraction, Transformation and Loading. The flow of the ETL process is, first it takes the data from the source system(s), second it cleans the data and finally the data will be transformed into the target system.[9]The operations being performed in the background of a data warehouse architecture ETL processes are responsible for them.

\section{Extraction}

[10]The data collected from different sources like internal and external sources are not usually integrated, incomplete and it may be duplicated. Therefore, the extraction process is required for collecting relevant and significant data from different source systems which are supporting in organization decision making. Prior to the transformation and cleansing process, the extracted data are sent to a temporary storage area which is called as Persistent Staging Area (PSA)

\section{Transformation}

Once the data arrives in the PSA, first it will be cleansed prior to physical storage in the targets.[11] The main function over the data which is being performed at this stage is data cleaning and noise reduction.Each time you want to convert incoming fields from your source system to InfoObjects on your BI InfoProviders, you create a dedicated TRANSFORMATION which consist one transformation rule for each object. To execute a transformation, Data Transfer Process (DTP) is used which you would create using the context menu on a transformation.

\section{Loading}

[12] This is the phase that loads the data into the targets of business warehouse which may vary and depend on the organization's requirement.[8]Data warehouse consists of PSA (Persistence Staging Area), Master data, DSO, Info Cube and Open hub destination. Initially, data is loaded in PSA.

\section{CONCLUSION}

$\mathrm{BI} /$ Data Warehousing tool set is a requirement for the continued success of your company. The Data Warehousing functionality if provided by the core component of SAP Net Weaver, BI. Business Intelligence tools and platform enable business to attain the maximum value from the information they collect. The information from external Data Sources, productive SAP applications can be integrated, transformed and consolidated in BI. To evaluate and interpret data, BI also provides the flexible reporting and analysis tools as well as facilitating data distribution.[13] Over the past decade, The Business Intelligence(BI) has evolved to rely progressively on real time data.BI systems auto initiate actions to the systems based on the rules and context to support many business processes.

In today's scenario, businesses and enterprise demand quick results.it is very much needed that along with the business analysis, the actions in response of the analysis results can be performed instantly. This can be achieved with the help of ETL process in Business Intelligence. Once the data is extracted from the various source systems it will be cleansed and transformed using transformation to the data targets with the help of data Transfer Process (DTP). When the data is available in the targets, reports can be generated from the data targets. In SAP BEx Designer the query can be created and executed.The paper explored the concept of BI and it objectives, Key components and features of BI and the ETL process.

\section{REFERENCES}

[1] S. AG, TBW10 - BI - Enterprise Data Warehousing, 2006.

[2] R. S. a. C. L. Golfarelli Matteo, Beyond Data Warehousing: What's next in Business Intelligence, 2004.

[3] P. S. V.Sujatha bai, "Business Application with Sap $\mathrm{Bi}$," International Journal of Computer Applications, 2012.

[4] "SAP Business Explorer," [Online]. Available: https://help.sap.com/saphelp_nw73ehp1/helpdata/en/5b/ 30d43b0527a17be10000000a114084/frameset.htm.

[5] P. Somasekaram, "Designing a Business Intelligence," 2013.

[6] S. A. TBW20, BI - Reporting \& Query Design, 2006.

[7] Z. Ren, "Building Business Intelligence Application with SAP BI," IEEE Xplore, 2009.

[8] S. N. S. T. Parul, "Performance Optimization for Extraction, Transformation, Loading and Reporting of data," IEEE Xplore, 2015.

[9] S. A. Vassiliadis P., "Extraction, Transformation, and Loading," Encyclopedia of Database Systems. Springer, 2009.

[10] N. Anand, "ETL and its impact on Business Intelligence," International Journal of Scientific and Research Publications, 2014

[11] D. R. J. Er. Sonal Sharma, "Modeling ETL Process in Data warehouse: An Exploratory Study Er. Sonal Sharma," IEEE Xplore, 2014.

[12] M. S. O. L. M. Y. Nur Alia Hamizah Mohamad Rodzi, "Significance of Data Integration and ETL in Business," IEEE Xplore, 2015.

[13] J. RANJAN, "BUSINESS INTELLIGENCE: CONCEPTS, COMPONENTS, TECHNIQUES AND BENEFITS," Journal of Theoretical and Applied Information Technology, 2009. 